\title{
Modelización espacial del hábitat potencial de la trufa negra (Tuber melanosporum Vittad.) en la provincia de Huesca (España)
}

\author{
R. Serrano-Notivoli1,* A. Incausa-Ginés², M. Martín-Santafé2, \\ S. Sánchez-Durán² y J.J. Barriuso-Vargas² \\ 1 Departamento de Geografía y Ordenación del Territorio. Universidad de Zaragoza, Pedro Cerbuna, \\ 12. 50.009 Zaragoza \\ 2 Centro de Investigación y Tecnología Agroalimentaria de Aragón (CITA), Avda. Montañana 930. \\ 50059 Zaragoza
}

\begin{abstract}
Resumen
El cultivo de la trufa negra (Tuber melanosporum Vittad.) es especialmente complicado por las exigencias ambientales que requiere. En el presente trabajo se propone un modelo de distribución de su hábitat en la provincia de Huesca (España). Para ello se usan factores condicionantes y limitantes de la presencia de la especie en el territorio en función de experiencias previas, y apoyado por un amplio conjunto de muestras de suelo tomadas directamente en campo. Se consideraron todas aquellas variables ambientales capaces de explicar la presencia/ausencia de la especie en el territorio. Dichas variables se clasifican en tres grupos según su naturaleza: climático, topográfico y edáfico. Se utilizaron Sistemas de Información Geográfica para integrar todas las variables ambientales en su dimensión espacial, y a través de un modelo de decisión de evaluación multicriterio se ponderaron para obtener una cartografía de aptitud. La asignación de los pesos relativos a cada variable se realizó en función de la importancia que tienen para el desarrollo de la especie, las experiencias similares en otros lugares, y en relación al área de estudio trabajada. La combinación de las variables ambientales que condicionan y limitan la aparición de la trufa negra en el territorio, en su dimensión espacial, dio como resultado una cartografía de distribución del hábitat potencial de la especie que muestra, a diferentes niveles, cuáles son las zonas con mayor o menor capacidad de producción de trufa negra. Los resultados muestran que un $2,75 \%$ de la superficie de toda la provincia tiene una aptitud óptima para el desarrollo de la especie en función de su hábitat potencial, cifra que se reduce hasta 1,62\% cuando se trata de zonas potencialmente cultivables. El mapa fue validado con las presencias de trufa conocidas disponibles para el área de estudio.
\end{abstract}

Palabras clave: Modelización, cartografía, SIG, evaluación multicriterio.

\begin{abstract}
A geospatial model for black truffle potential habitat (Tuber melanosporum Vittad.) in Huesca province (Spain)

Black truffle (Tuber melanosporum Vittad.) cultivation is especially complicated by their environmental requirements. We propose in this paper a distribution model of habitat for Huesca province (Spain). We used conditioning and restricting factors to the presence of the species in territory based on previous ex-
\end{abstract}

\footnotetext{
* Autor para correspondencia: rs@unizar.es
} http://dx.doi.org/10.12706/itea.2015.015 
periences, and supported by a broad collection of field samples. Environmental variables can explain the presence/absence of the species in the study area. These variables are classified in three groups according to their nature: climatic, topographic and edaphic. We integrated in a GIS all parameters to unify spatial resolution, and by a multi-criteria decision model weighted them to obtain suitability areas. The weights allocation were assigned in function of their importance to black truffle presence and previous experiences. The combination of environmental variables that are conditioning and restricting the presence of black truffle in territory, generates a cartography of potential habitat distribution at different levels These levels are areas with more or less production ability of black truffle. Results show that $2.75 \%$ of province has an optimal aptitude to species development, this number is reduced to $1.62 \%$ in potential cultivation. Map was validated with available presences of known truffle locations in study area.

Key words: Model cultivation, cartography, GIS, multicriteria evaluation.

\section{Introducción}

El cambio fundamental experimentado por la truficultura en España se produjo a partir de la década de los años 70. Es entonces cuando se incrementó el ritmo de despoblación del medio rural, se redujo el pastoreo, se abandonó el aprovechamiento silvícola, y los cultivos no mecanizables fueron colonizados por vegetación forestal. La consecuencia fue un incremento de la densidad vegetal en los montes, que también facilitó la iniciación y propagación de incendios forestales. Además, se realizaron repoblaciones forestales de coníferas en áreas no adecuadas que pertenecían a comunidades de quercíneas en general. Todo ello, unido a una gran sobreexplotación de las truferas, derivó en un descenso dramático de la producción silvestre española a partir de la década de los 80 (Reyna y García-Barreda, 2014).

Esta actividad productiva es compatible y complementaria con las más tradicionales del sistema agrario correspondiente. Además no se debe plantear como la única alternativa, sino como una diversificación de la economía rural, favoreciendo con ello un nuevo equilibrio territorial, manteniendo una población activa en estos espacios rurales que garantizan la preservación de sus paisajes. En la provincia de Huesca en los últimos 10 años se han llegado a implantar más de
1.000 ha de superficie trufera. La Administración local de la provincia ha facilitado este cultivo aportando más de 4 millones de euros para la plantación de árboles micorrizados y que empiezan a estar en plena producción (Gómez et al., 2013).

El cultivo de la trufa negra ha ido adquiriendo con el tiempo una mayor importancia en las zonas geográficas donde las características edáficas y climáticas son las adecuadas. En muchas regiones del arco mediterráneo, donde existe de forma natural esta especie de hongo, se encuentran zonas en las que no se conoce la presencia de trufa y que ofrecen condiciones edafoclimáticas óptimas o favorables para establecer el cultivo. Las dificultades existentes para identificar y localizar las zonas aptas para la producción de la trufa negra suponen un obstáculo para el fomento de esta actividad agroforestal en las regiones que ya son aptas para ello. Por otra parte, la determinación de los hábitats adecuados para el desarrollo natural de esta simbiosis hongoplanta conlleva el reconocimiento de los ecosistemas aprovechables para la truficultura.

Por estas razones, en España, han sido ya elaborados diferentes mapas de aptitud trufera, como son los de la Comunidad Valenciana (Domínguez et al., 2003), Albacete (Honrubia et al., 2006), Cataluña (Colinas et al., 2007), León (Rodríguez et al., 2008), Navarra (Gon- 
zález-Aramada, 2009) y algunas comarcas de la serranía de Cuenca (Aguirre et al., 2009). La metodología empleada se basa, en la mayor parte de los casos, en el uso de la cartografía disponible con carencia de aspectos edáficos que se resuelven mediante el uso de capas de series de vegetación, geológicas, litológicas o mediante localizaciones truferas conocidas de las que se infieren posteriormente sus características a modo de retroalimentación. Sin embargo esta cartografía resultante es, en la mayoría de los casos, de resolución espacial reducida y de un alcance limitado para la toma de decisiones. De esta forma se hace imprescindible la realización de nuevos mapas de regiones geográficas más reducidas y concretas que permitan una mayor definición de las superficies aptas o no para la truficultura, como puede ser el caso de la cartografía realizada en un monte privado en la zona de mont Ventoux en Francia (Chandioux et al., 2009).

En el presente trabajo se analizan los factores condicionantes y limitantes que afectan a la presencia de la trufa negra en la provincia de Huesca, un territorio de máxima importancia para el cultivo de Tuber melanosporum Vittad., ya que alberga en el entorno de la localidad de Graus (Somontano pirenaico) una de las zonas truferas de mayor producción mundial. Posteriormente se integra toda la información en un Sistema de Información Geográfica (SIG) para considerar la dimensión espacial, y por último se ponderan todas las capas de información en cada punto a través de métodos de evaluación multicriterio.

El objetivo final es el de obtener una cartografía del hábitat potencial de $T$. melanosporum en la provincia de Huesca. Además, se identifican las zonas más aptas para el cultivo en función de los usos del suelo restringidos dicho hábitat potencial. No se trata de definir dónde hay que cultivar la especie en la provincia, sino de determinar cuáles son las zonas más aptas únicamente en función de los requerimientos ambientales de la especie.

\section{Material y métodos}

Área de estudio

La provincia de Huesca (Figura 1) ocupa unos $15.600 \mathrm{~km}^{2}$ distribuidos en 10 comarcas que agrupan 202 municipios. La diversidad orográfica es caracterizada por la variedad de formas de relieve, desde las planicies de la Hoya de Huesca (125 m.s.n.m.), al pie de las Sierras Exteriores, hasta el Pirineo axial, zona central de disposición este-oeste del macizo montañoso con alturas superiores a los 3.000 metros en su límite superior (3.404 m.s.n.m. en el pico Aneto).

Su variedad altitudinal determina la presencia de diferentes tipos de clima, mediterráneo continentalizado en el sureste, donde se dan las temperaturas medias más elevadas; pasando por un ambiente de transición en la zona prepirenaica, donde van aumentando las precipitaciones a medida que se eleva la altitud, y las temperaturas medias van descendiendo; hasta el clima de montaña que se da en las zonas más elevadas, a partir de los 1.700-1.800 metros de altitud, donde la precipitación supera ampliamente los $1.200 \mathrm{~mm}$ anuales, en forma de nieve en invierno, y donde son muy frecuentes las heladas desde octubre hasta finales de mayo. En la Figura 2 se muestra la distribución mensual de la precipitación en el sector central de la provincia de Huesca. En comparación a la distribución Otoño-Invierno-Primavera-Verano que presentan las zonas truferas del Sistema Ibérico (Reyna, 2012), la precipitación estival en esta zona tiene mayor protagonismo que en las sierras ibéricas, lo que atenúa la mediterraneidad del clima y asegura mayor disponibilidad de agua durante el periodo crítico para el desarrollo de la trufa, fundamentalmente en la fase de producción del hongo.

En las zonas más elevadas (al norte) los suelos están muy poco desarrollados y dominan Leptosoles, Umbrisoles y Phaeozems, los Cam- 


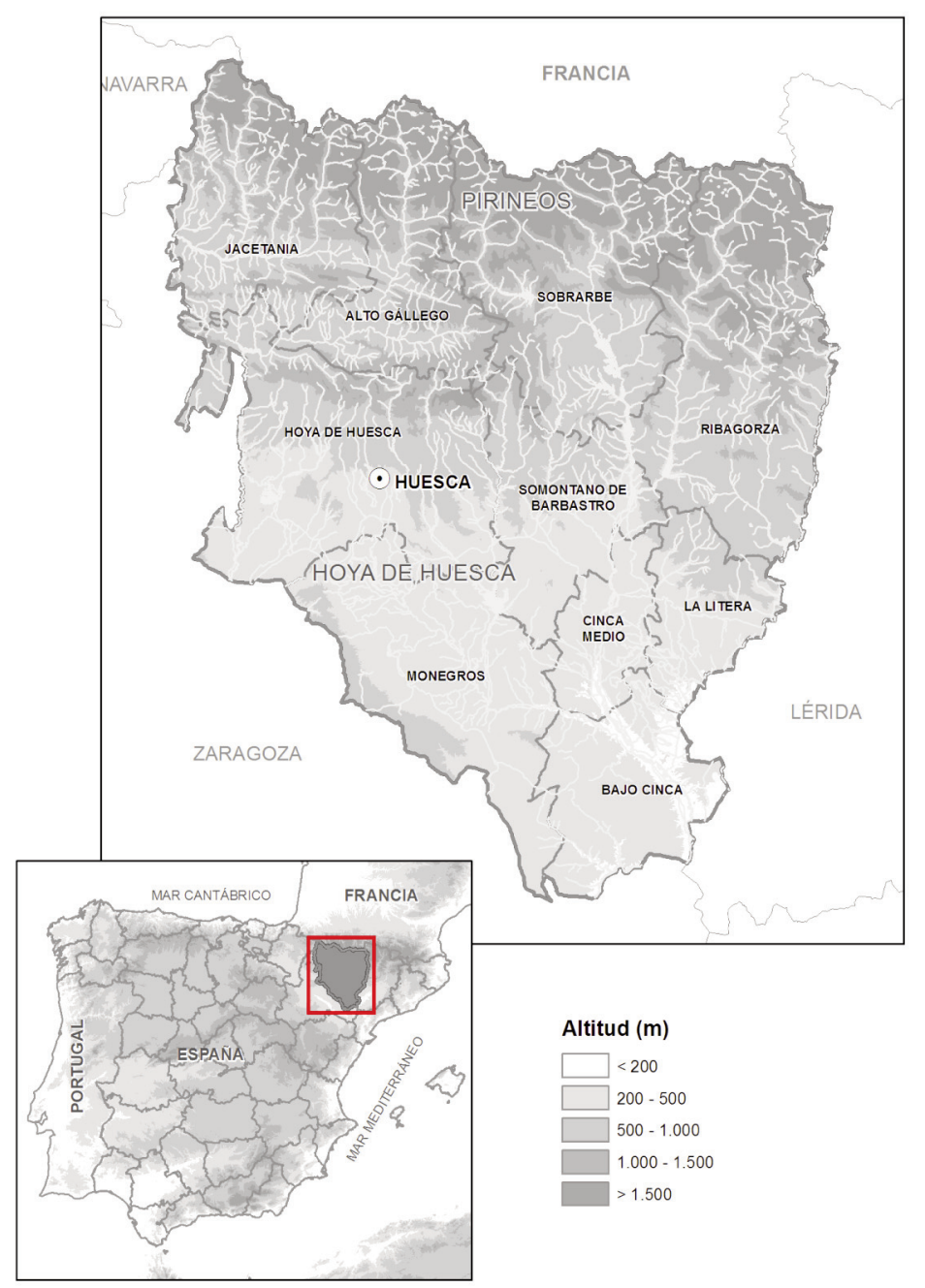

Figura 1. Área de estudio.

Figure 1. Study Area.

bisoles dominan el área prepirenaica, y el resto de la provincia está prácticamente cubierto por Regosoles y Calcisoles con otros tipos en menor medida.

La variedad de la vegetación oscense es muy amplia, fuertemente determinada por el clima y la altitud, en la mitad norte dominan los bosques de coníferas de alta montaña, y a menor altitud los bosques húmedos (hayedoabetales). Los bosques secos y marcescentes, sobretodo de encinar, marcan la transición a la depresión del Ebro, donde aparecen también, ya en las zonas bajas y más cálidas los pinares de Pinus halepensis y los sabinares. 


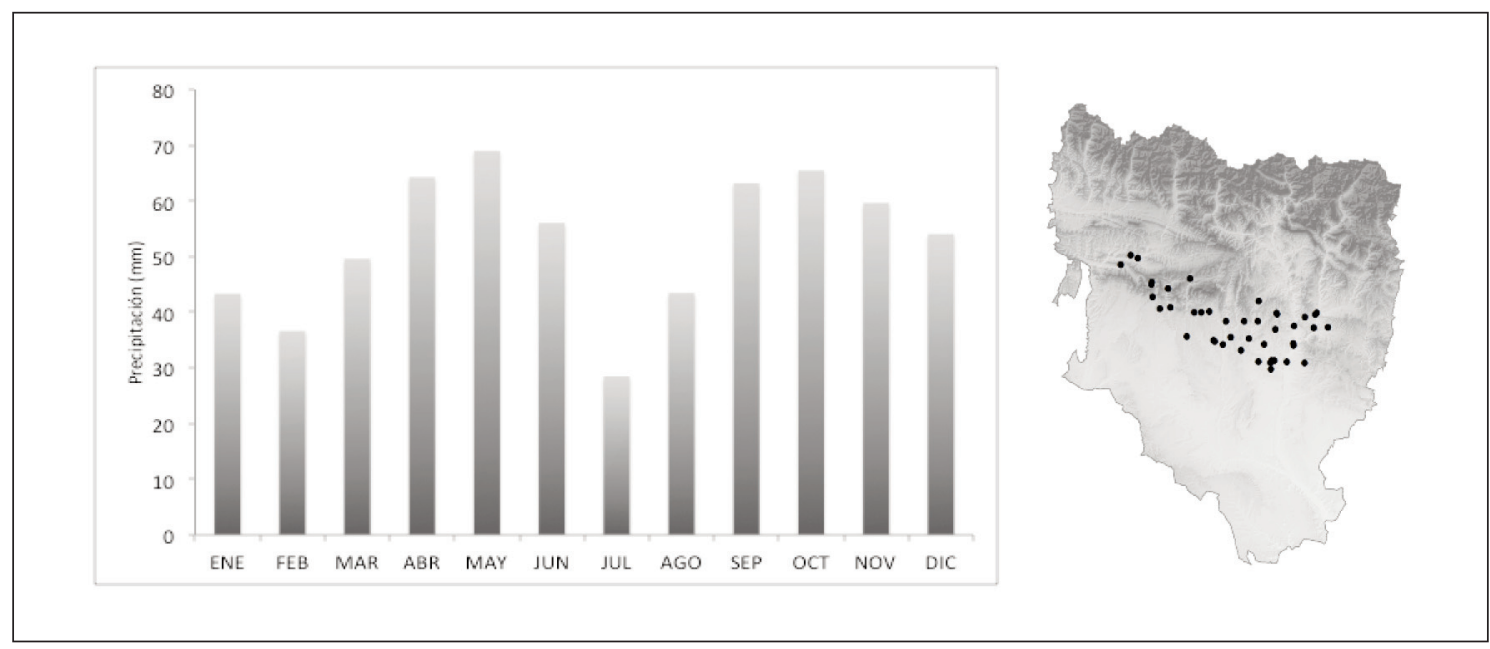

Figura 2. Distribución media mensual de la precipitación en 44 observatorios del sector central de la provincia de Huesca (Periodo 1971-2000).

Fuente: López et al. (2007).

Figure 2. Monthly mean distribution of precipitation at 44 observatories in central area of Huesca Province (Period 1971-2000).

Source: López et al. (2007)

Exigencias ambientales del cultivo de la trufa negra

La trufa negra requiere de ciertas condiciones ambientales que en conjunto determinan su presencia de manera tan exclusiva en algunas regiones o áreas del territorio. Para el presente trabajo, las exigencias ambientales se trataron como parámetros o variables de una ecuación que hay que resolver sobre un lugar del territorio, y cuyo resultado aporta la valoración potencial de esa área en la que la especie puede adaptarse con mayor o menor dificultad. Algunos de los parámetros ambientales que se utilizaron son limitantes porque excluyen la posibilidad de aparición de la especie en determinados lugares debido a sus características (altitud, precipitación total anual, temperatura media anual, temperatura del mes más cálido y temperatura del mes más frío), y otros son condicionantes ya que la posible aparición de la especie está condicionada a la magnitud o intensidad del parámetro ambiental presente en ese lugar (precipitación total estival, pendientes, etc.).

Los numerosos estudios sobre la distribución de la trufa negra (T. melanosporum) y otras especies de similares características biológicas en España (Domínguez et al., 2003; Honrubia et al., 2006; Colinas et al., 2007; Rodríguez et al., 2008; Aguirre et al., 2009; González-Aramada, 2009; Alonso-Ponce et al., 2010) además de otros sobre su cultivo (Reyna, 2012), muestran que la trufa depende de una serie de parámetros edáficos y climáticos básicos para el desarrollo y crecimiento de la especie en cualquier lugar. En el presente trabajo se escogieron aquellos parámetros o variables ambientales que son más determinantes para la presencia de la especie en el área de estudio, y se dividieron en tres grupos en función de su naturaleza (Colinas et al., 2007; Hall et al., 2008, Olivier et al., 2012, Reyna, 2012): i) Topográfico, ii) Climático, y iii) Edáfico. 
El grupo i) correspondiente a los factores topográficos engloba la altitud (ALT); la pendiente expresada en porcentaje (PEND); y una combinación de altitudes y orientaciones (ORI). Este grupo provoca que gran parte del territorio se considerase no apto para la aparición de la especie, principalmente por la altitud (altitudes óptimas entre 600 y 1.000 m.s.n.m.) y las fuertes pendientes (pendientes óptimas entre el 5 y el 15\%) (García-Barreda et al., 2012).

El segundo grupo ii) corresponde a los factores climáticos. La trufa negra está adaptada a un ambiente semiárido o mediterráneo (seco con tendencia continental) caracterizado por precipitaciones medias anuales de 600 a $900 \mathrm{~mm}$ que suelen concentrarse en primavera, final de verano y otoño (principalmente en forma de tormentas), contrastando con periodos secos en verano e invierno. Las variables incluidas en este grupo fueron la precipitación total anual (PTOT), que determina la presencia de la especie simbionte que alberga al hongo de la trufa negra, como la coscoja (Quercus coccifera L.) y el roble (Quercus robur L.), que presentan una ecología muy distinta (Reyna, 2012); la precipitación estival (PVER) suele ser más adecuada para el hábitat de la especie cuando se concentra con carácter mediterráneo (estacional, al principio del otoño y mitad de la primavera); la temperatura media anual (TMED), sin ser una variable climática demasiado indicativa en sí misma, revela un rango genérico en el que la planta es capaz de adaptarse. Reyna (2012) señala que el óptimo es entre 8 y $15^{\circ} \mathrm{C}$, lo que cubre prácticamente la mitad del territorio peninsular español eliminando las zonas de montaña; la temperatura media del mes de julio (TMAX) y la del mes de enero (TMIN) representan el máximo y el mínimo respectivamente de las medias del territorio, y sirven para establecer umbrales térmicos de adaptación.

El grupo iii) correspondiente a los factores edáficos es seguramente el de mayor rele- vancia, ya que establece las condiciones del medio directo en el que se ha de desenvolver la especie durante su crecimiento. Los suelos truferos son clasificados (Olivier et al., 2012) desde el punto de vista pedológico en tres tipos diferentes: Leptosols, suelos pardos calcáreos y suelos pardos cálcicos. Los suelos pardos calcáreos se encuentran incluidos entre los Calcosoles y los pardos calizos entre los Calcisoles. Aunque son los más habituales, no se pueden ignorar los Litosoles y los suelos coluviales, que se dan en truferas silvestres sin posibilidad de cultivo (Reyna, 2012). Como actualmente se carece de una información sobre distribución de los suelos completa para el área de trabajo y su estudio es inabordable para este objetivo, se optó por contemplar los parámetros edáficos de mayor importancia para la presencia de la especie. Éstos son el pH (PH), que ha de estar entre 7,5 y 8,5 ; la caliza activa (CAL), con valores inferiores al $30 \%$, la textura (TEX), que divide el territorio en función de la litología aflorante; y el contenido de materia orgánica (MAT) (Colinas et al., 2007; Hall et al., 2008, Olivier et al., 20012, Reyna, 2012).

\section{Información de partida}

Se introdujeron las variables ambientales divididas en tres grupos: parámetros climáticos, precipitación media anual, precipitación estival, temperatura media anual, media máxima anual, y media mínima anual; los topográficos, altitud, pendiente, y orientaciones; y los edáficos, caliza activa, textura, contenido en materia orgánica y $\mathrm{pH}$. La información climática proviene del Atlas Climático de Aragón (López et al., 2007) en formato ráster a una resolución espacial de $100 \mathrm{~m}$. La información edáfica se obtuvo mediante un total de 1.060 tomas de muestras en suelo arable a profundidades de entre 10 y $40 \mathrm{~cm}$ en zonas distribuidas por todo el territorio oscense, 434 de ellos aportados por la Diputación Provincial de Huesca, y 626 por el Laboratorio Agroambiental del 
Gobierno de Aragón. Las capas de: componentes texturales del suelo, caliza activa, y materia orgánica, se obtuvieron mediante la interpolación de la información contenida en los puntos correspondientes a los muestreos mediante Kriging Ordinario. La información topográfica sobre la altitud se obtuvo del MDE a $100 \mathrm{~m}$ de resolución espacial del Sistema de Información Territorial de Aragón (SITAR sitar.aragon.es), y de allí se derivaron los productos de pendientes y orientaciones mediante la herramienta Spatial Analyst de ArcMap 10.2. Toda la información sobre las variables necesarias para el desarrollo de la trufa en el territorio se integró en un SIG con el fin de analizarlas desde un punto de vista espacial adaptadas al área de estudio.

\section{Evaluación multicriterio}

Para modelizar espacialmente el hábitat potencial de la especie se siguió un modelo cartográfico de evaluación multicriterio (EMC) (Figura 3) (Romero, 1993; Barredo y Bosque, 1999; Malczewski, 1999; Gómez y Barredo, 2005). Se trata de un método de decisión en el que, junto con un SIG, los criterios (condicionantes y limitantes) se trabajan mediante variables ambientales adecuadamente reclasificadas y ponderadas. Las alternativas o variables utilizadas son definidas geográficamente en el sentido en el que los resultados del análisis dependen de su distribución espacial original (Malczewski, 1999). El grupo de agentes intervinientes en esta parte del

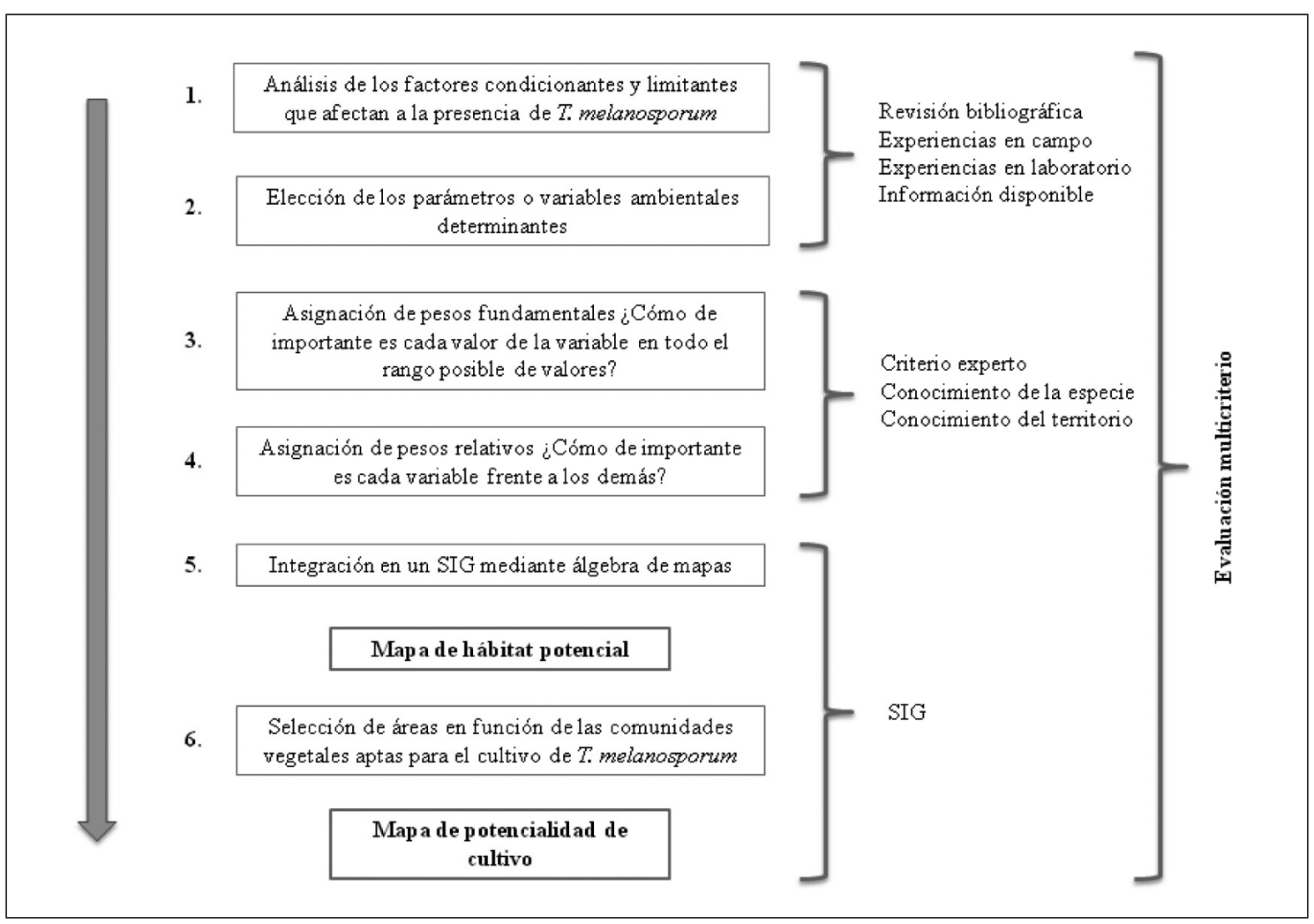

Figura 3. Fases y métodos en el proceso de obtención de las cartografías finales mediante evaluación multicriterio.

Figure 3. Methods and stages in map creation process by multi-criteria evaluation. 
proceso estuvo formado por expertos de varios ámbitos: el equipo de truficultura del Gobierno de Aragón (compuesto por biólogos, ingenieros de montes, ingenieros agrónomos, y geógrafos), además se tomaron opiniones de miembros de las asociaciones de truficultores de Aragón (en la provincia de Huesca), y de manera puntual de expertos en alguno de los ámbitos de la truficultura. En total se consultaron a más de 20 personas conocedoras del tema.

Se utilizaron doce variables o parámetros diferentes como determinantes de la producción de la trufa negra (descritos en el apartado 3.1 "Exigencias Ambientales"). Se considera que cada una de las variables posee un valor diferente en la determinación de la capacidad para la producción de trufa, lo que se denominó aptitud trufera.

Los parámetros utilizados responden a las exigencias ambientales enumeradas anteriormente, sin embargo, es necesario expresar en términos cuantitativos la importancia relativa de cada uno de los elementos para la posible presencia de la especie en cada lugar específico. La cuestión de asignar valores operables a los criterios utilizados ha sido discutida ampliamente (Barba y Pomerol, 1997; Gómez y Barredo, 2005; Colinas et al., 2007), y supone la intervención humana en casi todos los métodos utilizados. En general, la valoración debería estar basada en trabajos previos, clasificaciones establecidas para ciertas variables, análisis de los acontecimientos previos en el área de estudio, y otras fuentes que permitan llegar a una asignación sólida y justificada de los valores ordinales para las clases de cada criterio establecido (Gómez y Barredo, 2005). Para conseguir este objetivo se determinó su contribución en la realización de los mapas con dos ponderaciones o asignaciones de pesos relativos. La primera es una propiedad intrínseca a cada parámetro que está relacionada con los requerimientos correspondientes de esta variable en la producción natural de trufa negra, es decir la importancia relativa de ese valor concreto respecto a la presencia/ausencia de carpóforos de $T$. melanosporum en un paraje dado. La segunda está relacionada con el peso que tiene cada parámetro frente a los otros, es decir el peso relativo.

En este trabajo se utilizó el método de asignación directa, en el que se otorgan valores a cada criterio en función de su importancia para la presencia de la especie, siempre en base a la bibliografía existente y a la propia experiencia adquirida en los muestreos de campo. La falta de un criterio común o preestablecido por los investigadores y expertos en la materia es debido a la complejidad de la distribución de una especie tan escasa, y a la gran variabilidad de los parámetros observados en experiencias previas. Sin embargo, se considera que pueden existir unos parámetros estándar que pueden llegar a dar resultados, aunque no definitivos, si suficientes y útiles, y son los que se han utilizado en el presente trabajo.

Para establecer el rango de aptitud de cada parámetro en las capas del mapa final se hizo una revisión bibliográfica (Reyna et al., 2003; Colinas et al., 2007; Igor et al., 2010; Olivier et al., 2012) para adaptarlas a la realidad territorial de la provincia de Huesca. De esta manera, en la primera ponderación, se asignaron diferentes pesos a cada uno de los parámetros, estandarizándolos a su vez a una escala de 0 a 1 para que fueran comparables entre sí y poder operar matemáticamente con ellos. Esta ponderación es básica para encontrar las localizaciones óptimas del hábitat de la trufa, por lo que se realizó bajo los criterios ecológicos de la planta, que por otra parte son los requerimientos para su presencia.

Cuando se definieron los rangos de aptitud (Tablas 1, 2 y 3) se procedió a la priorización de los parámetros escogidos para la realización del mapa definitivo de aptitud trufera. Así, las altitudes por encima de 1.500 metros 
Tabla 1. Párametros del grupo climático evualuados y su ponderación en función de su importancia para la presencia de T. melanosporum

Table 1. Climatic group evaluated parameters and their weighting in function of their importance to T. melanosporum presence

\begin{tabular}{lrr}
\hline Parámetro & Rango de valores & Ponderación \\
\hline Precipitación total (PTOT) & $<400 \mathrm{~mm}$ & 0 \\
& $400-600 \mathrm{~mm}$ & 0,8 \\
& $600-900 \mathrm{~mm}$ & 1 \\
Precipitación estival (PVER) & $900-1.500 \mathrm{~mm}$ & 0,7 \\
& $>1.500 \mathrm{~mm}$ & 0 \\
Temperatura media anual (TMED) & $<50 \mathrm{~mm}$ & 0,1 \\
& $50-75 \mathrm{~mm}$ & 0,7 \\
& $75-185 \mathrm{~mm}$ & 1 \\
& $185-250 \mathrm{~mm}$ & 0,5 \\
& $>250 \mathrm{~mm}$ & 0,1 \\
Temperatura media mínima anual (TMIN) & $<8^{\circ} \mathrm{C}$ & 0 \\
& $8-10^{\circ} \mathrm{C}$ & 0,8 \\
& $10-15^{\circ} \mathrm{C}$ & 1 \\
& $15-18^{\circ} \mathrm{C}$ & 0,7 \\
& $>18^{\circ} \mathrm{C}$ & 0 \\
& $<17^{\circ} \mathrm{C}$ & 0,7 \\
& $17-24^{\circ} \mathrm{C}$ & 1 \\
& $>24^{\circ} \mathrm{C}$ & 0 \\
& $<1^{\circ} \mathrm{C}$ & 0,4 \\
\hline
\end{tabular}

actúan como un factor limitante, ya que es poco probable la presencia de masas continuas de simbiontes de la trufa a esta altitud en esta latitud, así como en las pendientes superiores al $60 \%$. Con una precipitación total anual por debajo de $400 \mathrm{~mm}$ y por encima de $1.500 \mathrm{~mm}$ también es improbable que aparezca la trufa negra, igual que con precipi- taciones en verano inferiores a $50 \mathrm{~mm}$ o superiores a $250 \mathrm{~mm}$. También las temperaturas pueden limitar el crecimiento, especialmente si de media anual está por debajo de los $8^{\circ} \mathrm{C}$ o por encima de los $18^{\circ} \mathrm{C}$, y más específicamente si las temperaturas más frías del año se dan por debajo de $1^{\circ} \mathrm{C}$ y las más cálidas por encima de $24^{\circ} \mathrm{C}$ de media. Ade- 
Tabla 2. Párametros evaluados en el grupo topográfico y su ponderación en función de su importancia para la presencia de $T$. melanosporum

Table 2. Topographic group evaluated parameters and their weighting in function of their importance to T. melanosporum presence

\begin{tabular}{lrr}
\hline Parámetro & Rango de valores & Ponderación \\
\hline Altitud (ALT) & $<200 \mathrm{~m}$ & 0 \\
& $200-400 \mathrm{~mm}$ & 0,2 \\
& $400-600 \mathrm{~mm}$ & 0,8 \\
& $600-1.000 \mathrm{~mm}$ & 1 \\
& $1.000-1.200 \mathrm{~mm}$ & 0,8 \\
& $1.200-1.500 \mathrm{~mm}$ & 0,2 \\
Pendiente (PEND) & $>1.500 \mathrm{~mm}$ & 0 \\
& $0-5 \%$ & 0,5 \\
& $5-15 \%$ & 1 \\
ORI ${ }^{1}$ & $15-30 \%$ & 0,8 \\
& $30-60 \%$ & 0,5 \\
& $(\mathrm{~S}, \mathrm{SE}, \mathrm{SO})>600 \mathrm{~m}$ & 0,2 \\
& $(\mathrm{~S}, \mathrm{SE}, \mathrm{SO})<600 \mathrm{~m}$ & 1 \\
& $(\mathrm{~N}, \mathrm{NE} . \mathrm{NO})>600 \mathrm{~m}$ & 0,5 \\
& $(\mathrm{~N}, \mathrm{NE} . \mathrm{NO})<600 \mathrm{~m}$ & 0,5 \\
& $(\mathrm{E}, \mathrm{O})$ & 1 \\
\hline
\end{tabular}

${ }^{1}$ combinación de orientación y altitud: S: sur; SE: sureste; SO: suroeste; N: norte; NE: noreste; NO: noroeste; E: este; O: oeste.

más, los factores edáficos también restringen buena parte del rango de valores previsible. Por una parte, el $\mathrm{pH}$ por debajo de 7,5 parece poco aceptable, aunque algún autor rebaja este dato a 7,2 (Estrada, 1987) o 7 (Estrada y Alcántara, 1990), así como el porcentaje de caliza activa por encima del $30 \%$. Aunque el parámetro más relevante en truficultura referente al calcio es el Ca intercambiable, es un parámetro indicativo de las necesidades de este elemento por la trufa ne- gra. (Colinas et al., 2007). El contenido en materia orgánica no es limitante, considerándose valores recomendables del 1 al $8 \%$ con un óptimo situado entre el 3 y $6 \%$ (Colinas et al., 2007), a diferencia de la textura del suelo, la cual es excluyente con una presencia elevada de arcillas, limos, o arenas en una proporción dominante de cualquiera de ellas.

La asignación de los pesos (columna "Ponderación" en las Tablas 1 a 3) se realizó en función de la importancia que tienen los pará- 
Tabla 3. Párametros evaluados en el grupo edáfico y su ponderación en función de su importancia para la presencia de T. melanosporum

Table 3. Edaphic group evaluated parameters and their weighting in function of their importance to T. melanosporum presence

\begin{tabular}{lcc}
\hline Parámetro & Rango de valores & Ponderación \\
\hline Caliza activa (CAL) & $<0,1 \%$ & 0 \\
& $0,1-30 \%$ & 0 \\
& $>30 \%$ & 1 \\
Textura (TEX) & TEX1* & 0,5 \\
& TEX2** & 0 \\
Materia orgánica (MAT) & TEX3*** & 0,8 \\
& $<2 \%$ & 1 \\
pH (PH) & $2-10 \%$ & 0,5 \\
\end{tabular}

* Franca; Franco-arcillosa-arenosa; Franco-arenosa; ** Arcillo-arenosa; Franco-arcillosa-limosa; Francoarcillosa; Franco-limosa; Arenosa-francosa; *** Arcillosa; Limosa; Arcillo-limosa; Arenosa; Areno-franca.

metros ambientales cuando adoptan valores que no son limitantes para la presencia de la especie. Por ejemplo, en la precipitación diversos autores coinciden en cuanto a valores medios óptimos, estableciendo la anual entre $600-900 \mathrm{~mm}$ y la estival en torno a 75-185 mm (Oliach et al., 2005; Sáez y De Miguel, 2008). Además existe una fuerte correlación entre pluviometría estival y producción de trufas (Ricard, 2003). Es por eso por lo que se asignaron valores correlativamente menores a medida que se alejan del óptimo establecido en otros trabajos. Para cada grupo de factores considerados se obtuvo un mapa de potencialidad (topográfica, climática, y edáfica).
La segunda ponderación se realizó según los parámetros que requiere la especie y según la adaptación al espacio sobre el que se quiere aplicar para obtener el mapa de hábitat potencial. En este trabajo se ponderaron 12 parámetros (Tabla 4) considerados determinantes para la producción de trufa negra. Otros autores han valorado un número diferente de variables, así Colinas et al. (2007) valoran 8, coincidentes con los propuestos en el presente trabajo. De esta manera se ponderó cada variable ambiental en función de la importancia que puede tener para el mapa de valoración final, y se sumaron todas ellas teniendo en cuenta sus pesos respecto al total. 
Tabla 4. Valores asignados a cada parámetro en la ponderación final Table 4. Values assigned to the parameters in the final weighting

\begin{tabular}{lcc}
\hline & \multicolumn{2}{c}{ Ponderación } \\
\hline Parámetro & De 0 a 1 & Sobre el total \\
\hline Altitud (ALT) & 0,75 & $10,20 \%$ \\
ORI $^{1}$ & 0,3 & $4,08 \%$ \\
Pendiente (PEND) & 0,4 & $5,44 \%$ \\
Temperatura media anual (TMED) & 0,4 & $5,44 \%$ \\
Temperatura media máxima anual (TMAX) & 0,5 & $6,80 \%$ \\
Temperatura media mínima anual (TMIN) & 0,4 & $5,44 \%$ \\
Precipitación total (PTOT) & 0,6 & $8,16 \%$ \\
Precipitación estival (PVER) & 0,85 & $11,56 \%$ \\
Textura (TEX) & 0,75 & $10,20 \%$ \\
pH (PH) & 1 & $13,61 \%$ \\
Caliza activa (CAL) & 1 & $13,61 \%$ \\
Materia orgánica (MAT) & 0,4 & $5,44 \%$ \\
\hline
\end{tabular}

${ }^{1}$ Combinación de orientación y altitud.

En función de las ponderaciones asignadas a cada parámetro, se estableció una suma ponderada [1]

$$
\overline{M F}=\frac{\sum_{i=1}^{n} v_{i} w_{i}}{\sum_{i=1}^{n} w_{i}}
$$

donde $\overline{M F}$ es el valor en el mapa final, calculado a partir del sumatorio de cada parámetro $\left(v_{i}\right)$ por su peso específico $\left(w_{i}\right)$, dividido entre la suma de todos los pesos asignados.

La suma ponderada se resolvió en un SIG mediante álgebra de mapas [2]

$\left(\left(\mathrm{ALT}^{*} 0.102\right)+(\mathrm{ORI} * 0.0408)+(\mathrm{PEND} * 0.0544)+(\mathrm{TMED} * 0.0544)+\left(\mathrm{TMAX}^{*} 0.068\right)\right.$

$+\left(\mathrm{TMIN}^{* 0.0544)}+\left(\mathrm{PTOT}^{*} 0.0816\right)+(\mathrm{PVER} * 0.1156)+(\mathrm{TEX} * 0.102)+\left(\mathrm{PH}^{*} 0.1361\right)\right.$

$\left.+(\mathrm{CAL} * 0.1361)+\left(\mathrm{MAT}^{*} 0.0544\right)\right) /(0.102+0.0408+0.0544+0.0544+0.068+0.0544+$

$0.0816+0.1156+0.102+0.1361+0.1361+0.0544)$ 
El análisis de sensibilidad de este método es dirigido, y está representado por las ponderaciones que se otorgan a cada variable en función de la importancia global que tienen para el desarrollo de la especie (determinado por las experiencias anteriores y trabajos desarrollados en otros lugares).

El mapa obtenido de esta forma se contrastó con una cartografía de hábitats originales para obtener como objetivo final el mapa de distribución de la potencialidad del cultivo de la trufa negra en la provincia de Huesca.

Una vez obtenido el mapa final de potencialidad de hábitat, se seleccionaron aquellas zonas que se consideran aptas para el cultivo en función de la vegetación presente. Es decir, eliminando del mapa de hábitat los bosques de árboles ectomicorrícicos, los cuales, siguiendo a Reyna et al. (2006) y a De Miguel et al. (2014) no suponen un precedente de uso del suelo adecuado para este cultivo independientemente de que se trate de masas productoras de trufa negra. Aceptando que los límites los marca la escala de trabajo (1:250.000), la extracción se realizó a partir de las categorías del Mapa Forestal de España 1:50.000 (MFE50) (Ministerio de Medio Ambiente) utilizando comunidades vegetales de coníferas, frondosas, encinares, enebrales, robledales y sabinares, principalmente. La superficie total supuso un $30 \%$ aproximadamente respecto al mapa de hábitat potencial.

Con la consecución del mapa de distribución potencial del cultivo no se pretende determinar las zonas en las que puede o no puede darse el cultivo de $T$. melanosporum en función de una simple categorización de usos del suelo, sino que se aporta una visión más específica del mapa de hábitat potencial de la especie a través de las experiencias en el entorno.
Validación

Es importante destacar que los modelos de validación están diseñados para los modelos de presencia-ausencia de especies (en este caso no se dispone de una información bien distribuida de presencias y ausencias en la zona de estudio, por eso se ha optado por otra metodología). El método aplicado en este trabajo permite la obtención de una cartografía del nicho potencial de T. melanosporum, el cual se ha resuelto mediante un método mecanicista en el que se han utilizado datos fisiológicos, estableciendo una relación causal entre la distribución de la especie y las variables que la determinan, independientemente de los registros de las especies en el territorio (Kearney y Porter, 2004 y 2009).

Aunque se espera que todos los métodos incluyan las presencias dentro de las zonas aptas, la realidad es que no todos los individuos ocupan los hábitats óptimos, ya que existen poblaciones en zonas marginales que luchan por sobrevivir en peores condiciones que los que se encuentran en el óptimo zonificado por las variables ambientales que las determinan. Está probado que es posible la aparición de especies en hábitats no adecuados para ella (Pulliam, 2000).

La única manera de evaluar la validez del método aplicado es la de superponer las 76 presencias disponibles de $T$. melanosporum en la provincia, provenientes del inventario de plantaciones de trufa negra del CITA con el mapa de hábitats resultante.

Para fortalecer la validación, se han integrado las mismas variables y presencias en un software de modelización de hábitats (ModEco) con el fin de obtener un gráfico ROC (que represente los verdaderos y falsos posi- 
tivos en función de los puntos integrados en el modelo). La replicación del proceso con ModEco se realizó con las mismas variables y con el modelo Bioclim (Beaumont et al., 2005) reservando un $20 \%$ de los puntos como validación. Este modelo determina teóricamente los límites probables de la distribución de la especie a través de variables ambientales similares. El área bajo la curva ROC (AUC) indica, para un punto de presencia y uno aleatorio seleccionados al azar, la probabilidad de que el valor de idoneidad previsto por el modelo para el punto de presencia sea mayor que el previsto para el punto aleatorio. Es una medida directa de la capacidad de discriminación del modelo, que toma valores próximos a 1 cuando existe un buen ajuste con los datos de evaluación, y cercanos a 0,5 cuando el ajuste no es mejor que el obtenido por azar (Benito y Peñas, 2007).

\section{Resultados}

La potencialidad climática (Figura 4.i) de la provincia de Huesca es muy buena en general, las zonas ocupadas por el Somontano Pirenaico y las Sierras Exteriores son las que obtuvieron mayor valoración principalmente por la distribución de las precipitaciones a lo largo del año, y por sus temperaturas, que no suelen alcanzar valores muy altos (por encima de $24^{\circ} \mathrm{C}$ de media máxima anual invalida la aptitud general del territorio). La distribución espacial de la valoración refleja la transición desde el clima mediterráneo húmedo de la zona sur del Somontano pirenaico hasta la influencia del clima de montaña en las zonas bajas de las Sierras Exteriores.

El mapa de aptitud edáfica (Figura 4.ii) muestra en general una aptitud buena para la presencia de la especie en todo el territorio no li-
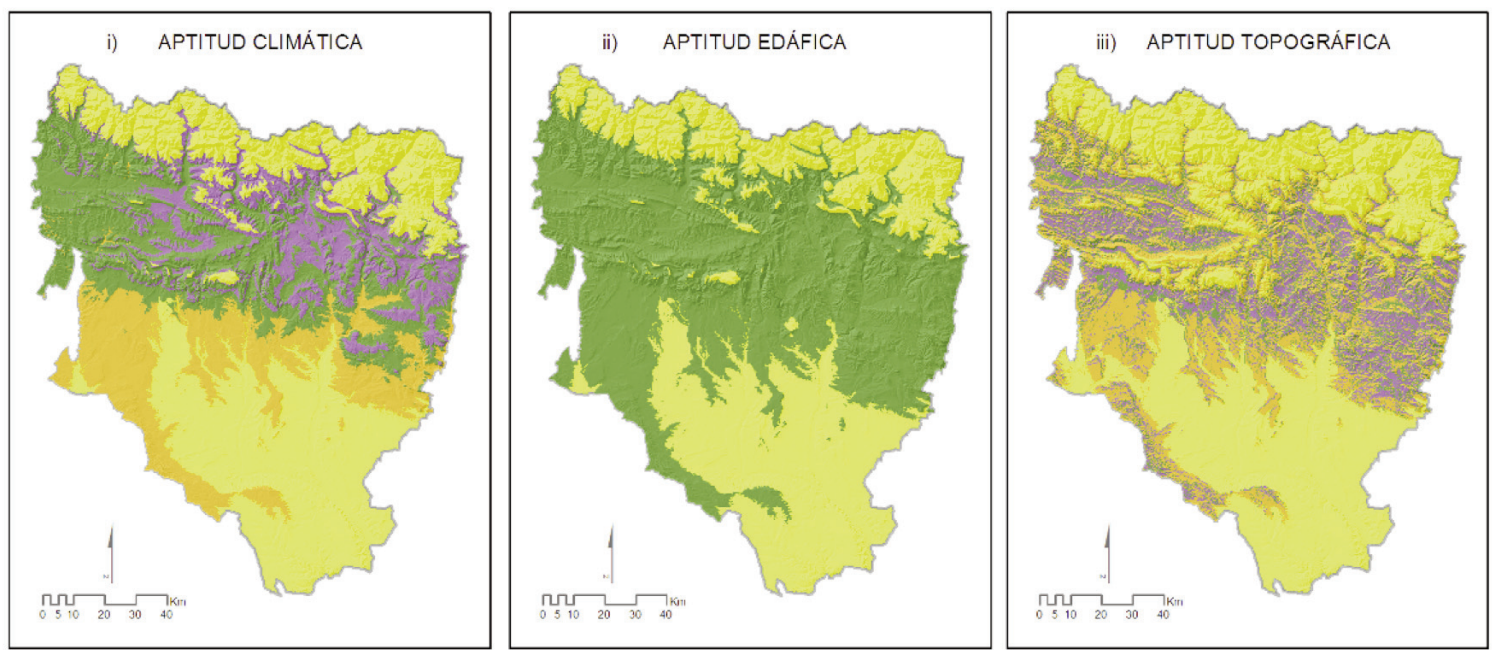

No Apto Baja Buena

Figura 4. Potencialidad climática, edáfica y topográfica.

Figure 4. Climatic, edaphic and topographic potentiality. 
mitado por otros factores. Tanto el pH como la caliza activa y la materia orgánica se encuentran en valores adecuados (entre 0,1 y 30 la caliza activa y entre 2 y 10 la materia orgánica), quizá tan solo la textura del suelo pueda modificar de manera local la aptitud de cada suelo.

La aptitud topográfica (Figura 4.iii) se muestra muy diversa, reflejando la heterogeneidad de la orografía oscense. En general en todas las zonas se observan valoraciones óptimas, matizadas por las orientaciones principalmente. Se eliminaron aquellas zonas por encima de $1.500 \mathrm{~m}$ y por debajo de $200 \mathrm{~m}$, lo que extrae del cálculo a gran parte del territorio.

En los tres mapas se observa una extensión en la mitad oeste del territorio que obtiene mejor valoración que el resto del sur de la provincia. Se debe por un lado a que se trata de una zona ligeramente más elevada que su entorno, con una combinación de orientaciones y altitudes favorable para la presencia de la trufa. Además, la Sierra de Alcubierre (ubicada al suroeste del área de estudio) dispone de las condiciones edáficas y climáticas aptas para la especie, junto con una masa forestal combinada con regiones de cultivo en secano, por lo que se configura como una región aceptable en cuanto a potencialidad de hábitat de la trufa negra se refiere.

El mapa final de hábitat potencial de la trufa negra (Figura 5.i) muestra en general una aptitud buena en todo el territorio no res-
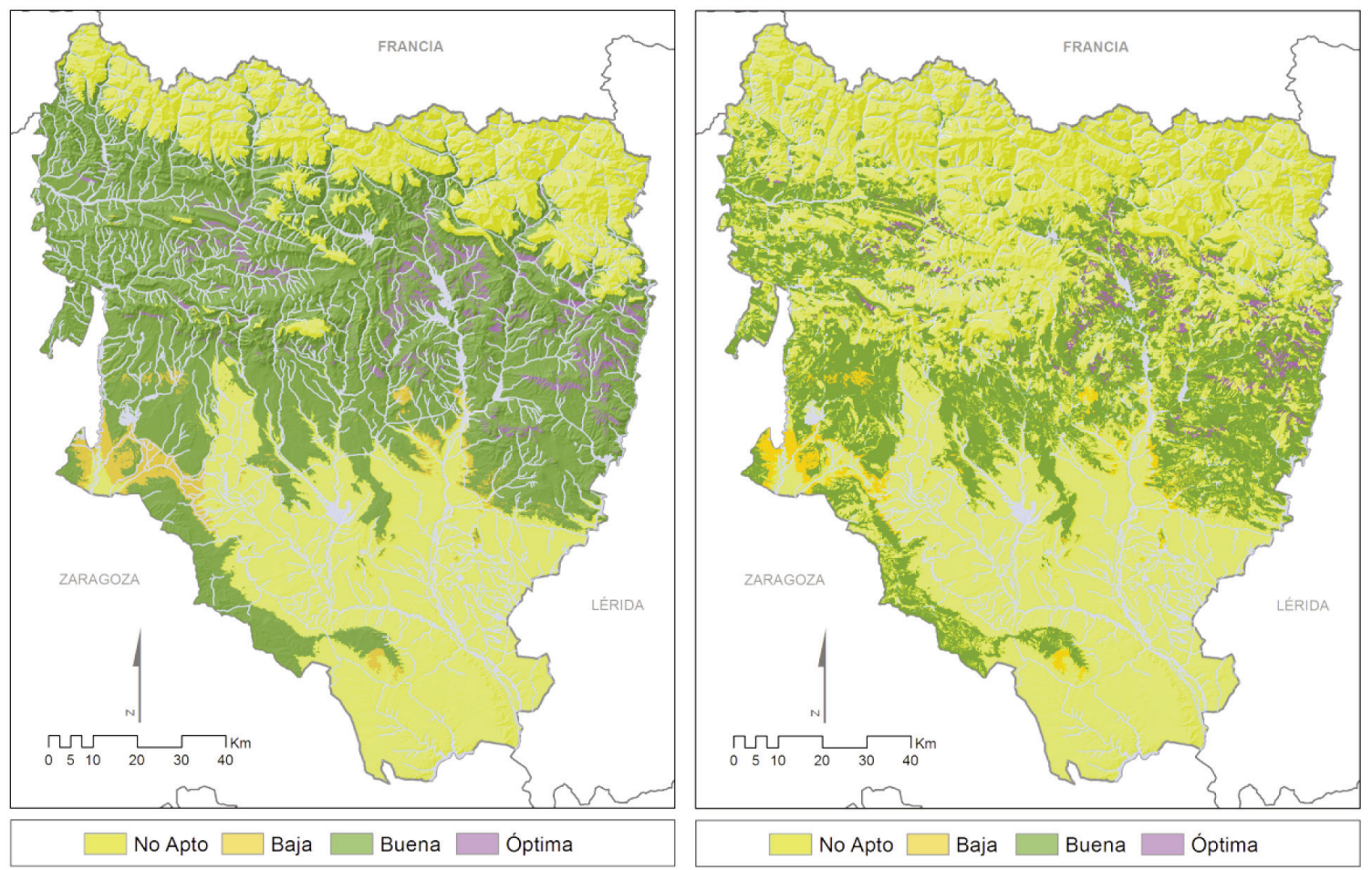

Figura 5. Mapa de hábitat potencial de la trufa negra (i) y Mapa de distribución de la potencialidad del cultivo (ii) en la provincia de Huesca.

Figure 5. Potential habitat map of black truffle (i) and cultivation potentiality map (ii) in Huesca province. 
tringido por las variables ambientales desde un principio. La ponderación final de cada uno de los parámetros ambientales utilizados produce un mapa que en absoluto es la media aritmética de los tres anteriormente mostrados (climático, edáfico y topográfico), sino que se trata de una modelización bastante cercana a la realidad de las zonas potenciales en las que la especie $T$. melanosporum es capaz de crecer en condiciones naturales.

En general, se muestra un territorio en el que se eliminan aquellas zonas demasiado elevadas y demasiado bajas topográficamente. La zona central de la provincia produce valores de aptitud buena (Figura 5.i) extendiéndose al ya mencionado apéndice occidental hasta la Sierra de Alcubierre. La matización de las zonas óptimas está principalmente en las orientaciones sur por encima de los 600 m.s.n.m., y se concentran en el espacio ocupado por el Somontano oriental de Barbastro, en la zona de Graus y el límite con Cataluña; más al norte en el entorno del embalse de Mediano; y en el oeste en los alrededores de Sabiñánigo y la cara norte del puerto del Monrepós, donde ya hay un cambio de la vegetación mediterránea desde la Hoya de Huesca hacia una de carácter más atlántico propia de las Sierras Exteriores. Las zonas No Aptas ocupan 668.518 hectáreas $(42,72 \%)$ en toda la provincia, las de categoría Baja $816.465,6$ ha $(52,18 \%)$, las de valoración Buena $36.776,1$ ha $(2,35 \%)$, y las zonas óptimas $42.904,2$ ha $(2,75 \%)$.

El mapa de distribución potencial del cultivo (Figura 5.ii) presenta mucha menos superficie (un $72,4 \%$ menos que la original) cubierta por zonas aptas para el cultivo. Este mapa muestra de manera más específica cuáles serían las zonas adecuadas en las que podría darse el cultivo de T. melanosporum. En esta nueva cartografía más detallada se muestran, previsiblemente, las zonas con mayor aptitud para el cultivo, sirviendo como modelo de gestión (No apta 71,03\%, Baja 1,38\%, Buena $25,97 \%$, Óptima $1,62 \%$, respecto a la superficie total del área de estudio).
La validación (Figura 6) indica que todas las observaciones se corresponden con áreas consideradas dentro del hábitat potencial según el modelo, estando la mayoría de ellas $(94,3 \%)$ en zonas consideradas Buenas, y una pequeña parte $(5,3 \%)$ en zonas Óptimas. En el mapa de potencialidad de cultivo un $68,4 \%$ de los puntos se corresponden con zonas Buenas, un 5,3\% en zonas Óptimas, y un $26,3 \%$ aparecen en zonas No Aptas lindando con zonas de distribución marginal.

Los resultados muestran un valor de AUC (Área Bajo la Curva) de 0,86. Este valor indica, para un punto de presencia y uno aleatorio seleccionados al azar, la probabilidad de que el valor de idoneidad previsto por el modelo para el punto de presencia sea mayor que el previsto para el punto aleatorio. La robustez del modelo viene determinada por la obtención de valores superiores a 0,85 (Marmion et al., 2009).

\section{Discusión y conclusiones}

La trufa negra es una especie con unos requerimientos ambientales muy específicos. La metodología de EMC aplicada es idónea para este tipo de modelización, ya que no existe un modelo general aplicable a la especie, sino que los parámetros ambientales utilizados han de adaptarse a cada territorio. Otros autores han utilizado metodologías basadas en la información multivariante para estimar la probabilidad de la aparición de la trufa negra en el territorio según las propiedades existentes en el suelo (Castrignanò et al., 2000). Aún teniendo en cuenta los rangos generales de cada variable en los que se mueve la especie, es importante tener un conocimiento profundo del territorio, ya que es el que mostrará las aptitudes de cada parte del mismo. Por tanto es necesaria una intervención humana en los procesos de decisión, especialmente en la asignación de pesos a cada parámetro utilizado. Establecer un modelo 


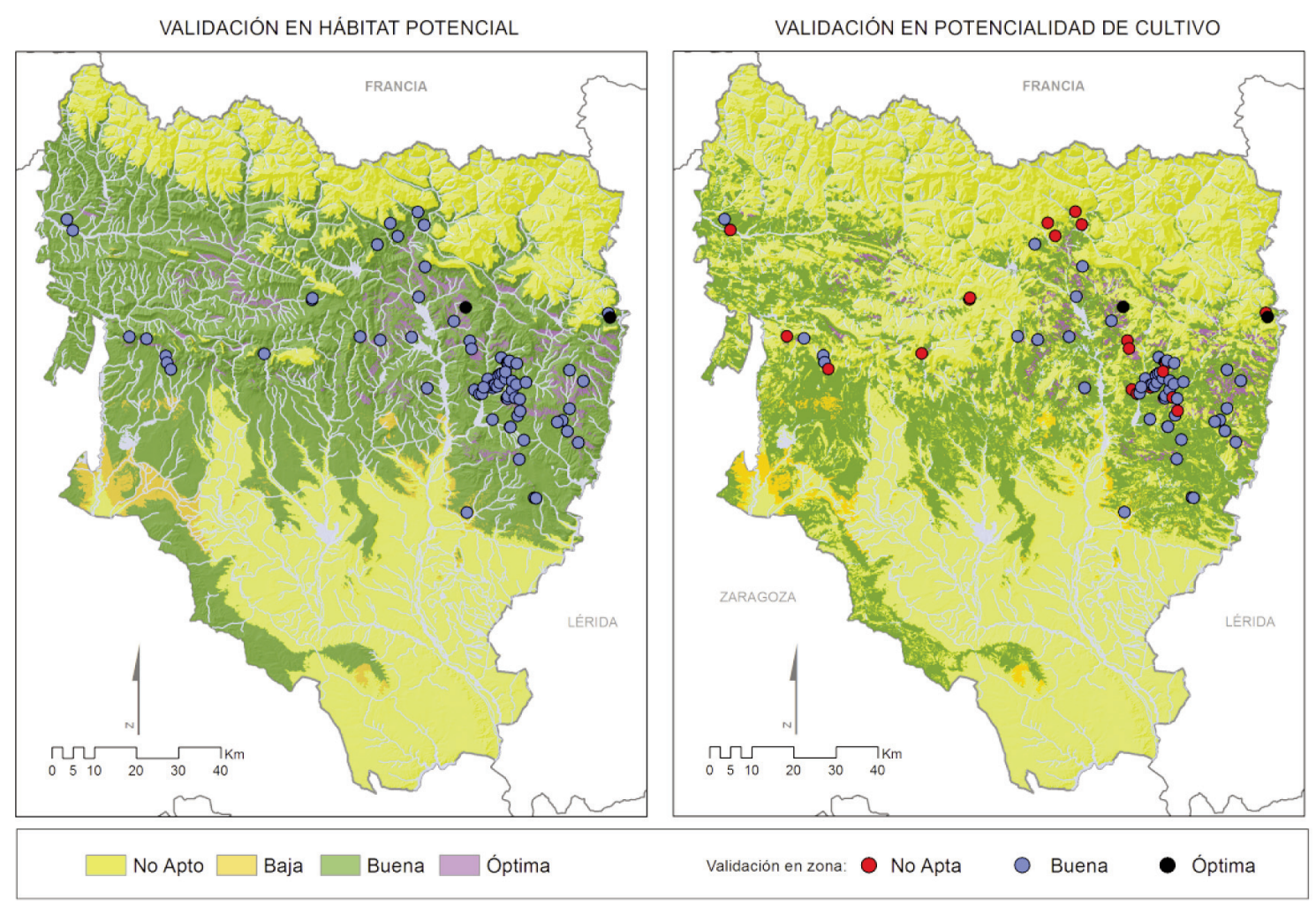

Figura 6. Ubicación de los puntos de cultivo actual en los mapas de hábitat potencial y potencialidad de cultivo.

Figure 6. Location of current crops in potential habitat map and cultivation potentiality map.

de hábitat para regiones tan amplias como la provincia de Huesca, e incluso de mayor tamaño, requiere de la laxitud de los condicionantes de presencia, esto es, hay que establecer rangos de valores en cada parámetro que se adapten a la escala de trabajo, y a los datos disponibles en el momento de elaborar el modelo.

Aragón es una región conocida por su producción trufera desde mediados del siglo pasado. La recolección profesional en truferas silvestres es una práctica habitual realizada por agricultores de la Comunidad Autónoma. La información recogida entre estos cazadores de trufa referente a las zonas o Comarcas con producción de trufa silvestre es la que ha llevado a considerar un sistema de validación de este mapa de aptitud para el cultivo de la trufa. Esta consideración junto con la experiencia derivada de la implantación de más de 1.000 ha de truferas cultivadas en zonas inicialmente apropiadas para el cultivo de este hongo en la provincia de Huesca, indica el grado de acierto de los resultados de esta cartografía con la realidad tal y como se indica en el apartado de validación. Aunque la resolución final ha sido de 100 metros, los resultados no intentan transmitir una definición de tal alcance para el territorio, pero sí que sirven para matizar determinadas zonas de aptitud óptima que se encuentran en 
zonas de borde de los límites de distribución de los parámetros de aptitud, especialmente en zonas de cambios orográficos bruscos.

La ventaja de desarrollar un modelo de distribución de hábitat para una zona concreta del territorio es que para estudiar una posible evolución del mismo solamente es necesario modificar los valores de los parámetros que lo integran. Hoy en día, las únicas variables que tienen la capacidad de ser predichas con un margen de confianza significativo son las climáticas. Las predicciones anunciadas por el Panel Intergubernamental de Cambio Climático - IPCC (IPCC, 2013) muestran tendencias de incremento de temperaturas y con ellas cambios importantes en las precipitaciones a escala regional, variando según las zonas. En este sentido algunos autores ya apuntan a la alteración de la distribución geográfica de algunas especies de trufa como la de verano (Tuber aestivum Vittad.) (Splivallo et al., 2012). Parece lógico pensar que estos cambios en los hábitats de la trufa negra se van a producir y de hecho se están produciendo, por lo que es importante obtener modelos regionales para las especies que permitan estudiar cómo evolucionarán las zonas óptimas en el futuro, lo que facilitará en gran medida la gestión. No obstante, la incertidumbre de los modelos climáticos a futuro, unida a la incertidumbre propia de los modelos de distribución de hábitats desarrollados con cualquier método disponible, hacen que la previsión de cambio en las zonas de distribución sea muy incierta y requiera de estudios más amplios y con buenos datos de partida.

La combinación de las variables ambientales que condicionan y limitan la aparición de la trufa negra ( $T$. melanosporum) en el territorio, en su dimensión espacial, produce una cartografía de distribución del hábitat potencial de la especie que muestra, a diferentes niveles, cuales son las zonas con mayor o menor aptitud.
Los resultados obtenidos muestran que:

- La combinación de los métodos de evaluación multicriterio EMC a través de las herramientas SIG son las más idóneas tanto por su facilidad de uso como por su versatilidad a la hora de manejar un conjunto de variables ambientales.

- Los parámetros ambientales que se introducen en el modelo han de ser todos aquellos que sean capaces de explicar la presencia/ausencia de la especie en el territorio. La asignación de los pesos de cada uno de ellos ha de hacerse en función de criterios rigurosos con la realidad, y en relación al área de estudio trabajada.

- La cartografía resultante en la provincia de Huesca muestra la aproximación a una distribución potencial de la trufa negra, calificando diferentes zonas en función de sus aptitudes naturales para la posible aparición de la especie.

\section{Bibliografía}

Aguirre JL, Díaz G, Bustamante B, Canales M, Aparicio A, Talabante C, Larrán A (2009). Estudio de la potencialidad de la truficultura en las comarcas de Molina de Aragón-Alto Tajo y Serranía de Cuenca. Universidad de Alcalá. 55 p.

Alonso-Ponce R, Águeda B, Ágreda T, Modrego MP, Aldea J, Martínez-Peña F (2010). Un modelo de potencialidad climática para la trufa negra (Tuber melanosporum) en Teruel (España). Forest Systems 19(2): 208-220.

Barba S, Pomerol J CH (1997). Decisiones multicriterio. Fundamentos teóricos y utilización práctica. Alcalá de Henares. Servicio de Publicaciones de la Universidad de Alcalá. 420 p.

Barredo Jl, Bosque J (1999). Multicriteria evaluation methods for ordinal data in a GIS environment. Geographical Systems 5, 313-327. 
Beaumont LJ, Hughes L, Pulsen M (2005). Predicting species distributions: use of climatic parameters in BIOCLIM and its impact on predictions of species' current and future distributions. Ecological Modelling 186, 250-269.

Benito B, Peñas J (2007). Aplicacion de modelos de distribucion de especies a la conservacion de la biodiversidad en el sureste de la Peninsula Iberica. GeoFocus 7: 100-119.

Castrignanò A, Goovaerts P, Lulli L, Bragato G (2000). A Geostatistical approach to estimate probability of occurence of Tuber melanosporum in relation to some soil properties. Geoderma 98, 95-113.

Chandioux O, Moundy P-J, Diette S, (2006). Cartographie du potentiel de production de truffes en milieu naturel. Mise au point d'une méthode adaptée a la foret privée du mont Ventoux. Revue Forestière Française LXI · 629-642.

Colinas C, Capdevila JM, Oliach D, Fischer CR, Bonet JA (2007). Mapa de aptitud para el cultivo de la trufa negra (Tuber melanosporum Vitt.) en Cataluña. Centro tecnológico forestal de Cataluña. Solsona. 134 p.

De Miguel AM, Águeda B, Sánchez S, Parladé J (2014). Ectomycorrhizal fungus diversity and community structure with natural and cultivated truffle hosts: applying lessons learned to future truffle culture. Mycorrhiza 24 (Suppl. 1): S5-S18.

Domínguez JA, López C, Rodríguez JA, Saiz JA (2003). Caracterización de rodales truferos en la Comunidad Valenciana. Ecología 17, 181-190.

Estrada JM (1987). La trufa y la realidad de su cultivo. El cultivo racional de la trufa. El Cultivador moderno 809, 62-65.

Estrada JM, Alcántara C (1990). La tòfona. Ed. Servicio de Extensión Agraria del Departamento de Agricultura, Ganadería y Pesca. Generalitat de Catalunya, Barcelona, $26 \mathrm{p}$.

García-Barreda S, Reyna S, Pérez-Badía R, Rodríguez-Barreal JA (2012). Ecología de la trufa y las áreas truferas. Cap. 5. Truficultura. Fundamentos y técnicas. Ed. Mundi-Prensa, Madrid, 153-208.
Gómez M, Barredo JI (2005). Sistemas de información geográfica y evaluación multicriterio en la ordenación del territorio Ed. RA-MA, Madrid.

Gómez E, Martín M, Sánchez S, Betorz G, Puig-Pey M, Barriuso J (2013). Evolution of black truffle culture in the province of Huesca (Spain). En $1^{\text {st }}$ International Congress of Trufficulture Tuber 2013, Teruel (España), 5-8 Marzo.

González-Aramada B. (2009). Delimitación de áreas de producción potencial de trufa negra (Tuber melanosporum Vitt.) en Navarra mediante GIS. Proyecto fin de carrera. Universidad Pública de Navarra. 157 p.

Hall IR, Brown GT, Zambonelli A, (2008). Taming the truffle. The History, Lore, and Science of the Ultimate Mushroom. Timber Press. Portland, Oregon EE. UU. 303 p.

Honrubia M, Fernández A, Moya D, González A, de las Heras J (2006). Potencialidad de la trufa negra (Tuber nigrum Bull.) en la provincia de Albacete. Montes 83, 35-40.

Igor B, Matteo G, Paolo R (2010). Carte di attitudine dei suoli ai tartufi in Piemonte metodologie e risultati. Atti 3er Congresso Internazionale de Spoleto sul Tartufo Spoleto 2008: 227-239.

IPCC (2013). Climate Change (2013). The Physical Science Basis. Contribution of Working Group I to the Fifth Assessment Report of the Intergovernmental Panel on Climate Change (Eds. Stocker TF, Qin D, Plattner GK, Tignor M, Allen SK, Boschung J, Nauels A, Xia Y, Bex V, Midgley P). Cambridge University Press, Cambridge, Reino Unido y Nueva York, EE. UU. 1535 p.

Kearney M, Porter WP (2004). Mapping the fundamental niche: physiology, climate, and the distribution of a nocturnal lizard. Ecology 85, 3119-3131.

Kearney M, Porter WP (2009). Mechanistic niche modelling: combining physiological and spatial data to predict species ranges. Ecology Letters $12,334-350$.

López F, Cabrera M, Cuadrat JM (2007). Atlas Climático de Aragón. Departamento de Medio Ambiente. Gobierno de Aragón. 222 p.

Malczewski J (1999). GIS and multicriteria decision analysis. Nueva York, J. Wiley, 392 p. 
Marmion M, Parviainen M, Luoto $M$, Heikkinen RK, Thuilier W. (2009). Evaluation of consensus methods in predictive species distribution modelling. Diversity and Distribution 15: 59-69.

Oliach D, Bonet JA, Fischer CR, Olivera A, Martínez de Aragón J, Suz LM, Colinas C (2005). Guia tecnica para el cultivo de trufa negra (Tuber melanosporum Vitt.). Ed. Centre Tecnologic Forestal de Catalunya. $30 \mathrm{p}$.

Olivier JM, Savignac JC, Sourzat P (2012). Truffe et Trufficulture. Ed. FANLAC, Périgueux, France. $398 \mathrm{p}$.

Pulliam HR (2000). On the relationship between niche and distribution. Ecology Letters 3, 349: 361.

Reyna S, de Miguel A, Palazón C, Hernández A, de Román M (2003). Truffle cultivation in Spain: state of the art and future prospects. $3^{\text {rd }}$ International Workshop on Edible and Mycorrhizal Mushrooms, University of Victoria, British Columbia, Canada.

Reyna S, García-Barreda S, Folch L (2006). Influencia del uso del suelo sobre el potencial de inóculo ectomicorrícico y la competitividad de las ectomicorrizas de Tuber melanosporum: evaluación mediante bioensayos en invernadero. Investigación Agraria: Sistemas Recursos Forestales 15(3): 308-320.
Reyna S (2012). Truficultura. Fundamentos y técnicas. Ed. Mundi-Prensa, Madrid, 720 p.

Reyna S, García-Barreda S (2014). Black truffle cultivation: a global reality. Forest Systems 23(2): 317-328.

Ricard JM (2003). La truffe. Guide technique de trufficulture. Ed. Centre technique interprofessionnel des fruits et légumes, París, $268 \mathrm{p}$.

Rodríguez JR, Acedo C, Marabel M, Álvarez MF (2008). Localización mediante SIG de zonas potencialmente truferas en la provincia de León. Uned. Espacio, Tiempo y Forma. Serie VI, Nueva época. Geografía, $n^{\circ} 1,83-91$.

Romero C (1993). Teoría de la Decisión Multicriterio: Conceptos, Técnicas, y Aplicaciones. Madrid. Alianza.

Sáez R, de Miguel AM (2008). La trufa. Guía de truficultura, ITGA-Universidad de Navarra, Pamplona, $132 \mathrm{p}$.

Splivallo R, Rittersma R, Valdez N, Chevalier G, Molinier V, Wipf D, Karlovsky P (2012). Is climate change altering the geographic distribution of truffles? Frontiers in Ecology and the Environment 10(9): 461-462.

(Aceptado para publicación el 24 de octubre de 2014) 